\title{
COVID-19: Examining the case growth-rate due to Visitor vs Local Mobility using Machine Learning: A Study in the United States
}

satya katragadda

ravi teja bhupatiraju

vijay raghavan

ziad ashkar

raju gottumukkala ( $\sim$ gnraju@ieee.org )

\section{Research Article}

Keywords: COVID-19, mobility, visitor risk, machine learning

Posted Date: September 20th, 2021

DOI: https://doi.org/10.21203/rs.3.rs-916701/v1

License: (c) (1) This work is licensed under a Creative Commons Attribution 4.0 International License.

Read Full License 


\section{RESEARCH}

COVID-19: Examining the case growth-rate due to ${ }^{3}$ Visitor vs Loçal mobility using Machine Learning: : A Study in the United States

Satya Katragadda, Ravi Teja Bhupatiraju, Vijay Raghavan, Ziad Ashkar and Raju Gottumukkala* 
1

\section{Abstract}

Background: Travel patterns of humans play a major part in the spread of infectious diseases. This was evident in the geographical spread of COVID-19 in the United States. However, the impact of this mobility and the transmission of the virus due to local travel, compared to the population traveling across state boundaries, is unknown. This study evaluates the impact of local vs. visitor mobility in understanding the growth in the number of cases for infectious disease outbreaks.

Methods: We use two different mobility metrics, namely the local risk and visitor risk extracted from trip data generated from anonymized mobile phone data across all 50 states in the United States. We analyzed the impact of just using local trips on infection spread and infection risk potential generated from visitors' trips from various other states. We used the Diebold-Mariano test to compare across three machine learning models. Finally, we compared the performance of models, including visitor mobility for all the three waves in the United States and across all 50 states.

Results: We observe that visitor mobility impacts case growth and that including 21 visitor mobility in forecasting the number of COVID-19 cases improves prediction accuracy by $34 \%$. We found the statistical significance with respect to the performance improvement resulting from including visitor mobility using the Diebold-Mariano test. We also observe that the significance was much higher during the first peak March to June 2020.

Conclusion: With presence of cases everywhere (i.e. local and visitor), visitor mobility (even within the country) is shown to have significant impact on growth in number of cases. While it is not possible to account for other factors such as the impact of interventions, and differences in local mobility and visitor mobility, we find that these observations can be used to plan for both reopening and limiting visitors from regions where there are high number of cases.

Keywords: Interval vs External Mobility; COVID19 Forecasting; Machine Learning 
${ }^{1} 1$ Introduction ${ }^{1}$

${ }^{2}$ COVID-19 has spread rapidly around the world, nearing 174 million confirmed cases ${ }^{2}$

${ }^{3}$ and more than 3.75 million deaths reported globally as of June 09, 2021 (John $^{3}$

${ }^{4}$ Hopkins University, 2020). 4

5 Many countries have passed the first and second peak, and aggressive vaccination ${ }^{5}$

${ }^{6}$ efforts and containment measures have limited the spread of the pandemic. While ${ }^{6}$

${ }^{7}$ counties are beginning to slowly reopen, the threat from the pandemic is far from ${ }^{7}$

${ }^{8}$ over, especially as the new delta variant that has spread to multiple countries. $\mathrm{A}^{8}$

${ }^{9}$ question remains as to the risk contribution of external visitors, as rebound travel ${ }^{9}$

${ }^{10}$ begins as cases come down and restrictions begin to ease. 10

11 The importance of tracking human mobility as a significant indicator to un- ${ }^{11}$

${ }^{12}$ derstand and predict the spread of COVID-19 has been an active research topic ${ }^{12}$

${ }^{13}[1,2,3,4,5,6,7]$. Researchers and local governments continue to track human ${ }^{13}$

${ }^{14}$ mobility in their communities through anonymized cell phone data made available ${ }^{14}$

${ }^{15}$ through various data providers $[8,9,10]$. An earlier study by Badr et al found $\mathrm{a}^{15}$

${ }^{16}$ strong linear correlation between mobility ratio and COVID-19 growth rate between ${ }^{16}$

${ }^{17}$ Jan 24th, 2020, and April 17, 2020, for the top 20 US counties that had the highest ${ }^{17}$

${ }^{18}$ number of cases [11]. Several studies in United States, Europe, and China report ${ }^{18}$

${ }^{19}$ association between mobility and stay-at-home orders with growth in number of ${ }^{19}$

${ }^{20}$ cases $[12,13,14,15,16,17,18,19,20]$. More recent work also studied the impact ${ }^{20}$

${ }^{21}$ of lockdowns on mobility [21].

${ }^{22}$ The visitor mobility has also been studied in different contexts. For instance ana- ${ }^{22}$

${ }^{23}$ lyzed tourist/visitor demand to various destinations to estimate potential COVID- ${ }^{23}$

2419 risk exposure $[22,23,24]$. However, there are no studies to analyze the difference 24

${ }^{25}$ between visitor and local mobility. Linka et al. used a global network mobility model ${ }^{25}$

${ }^{26}$ with a local epidemiology model to simulate and predict the COVID-19 outbreak ${ }^{26}$

${ }^{27}$ across Europe [19]. They have shown that mobility network of air travel can pre- ${ }^{27}$

${ }^{28}$ dict the global diffusion pattern of a pandemic and unconstrained mobility could ${ }^{28}$

${ }^{29}$ accelerate the spread of COVID-19 in Europe, using a latent period of 2-6 days and ${ }^{29}$

${ }^{30}$ infectious period or 3 to 18 days. Kraemer et al, used real-time mobility data from ${ }^{30}$

${ }^{31}$ Wuhan and detailed travel history to elucidate the role of case transmission in cities ${ }^{31}$

${ }^{32}$ across China [25]. They found that special distribution of COVID-19 cases in China ${ }^{32}$

${ }^{33}$ and the magnitude of the early epidemic (total number of cases until 10 February ${ }^{33}$ 
${ }^{1} 2020$ ) outside of Wuhan was very well predicted by the volume of human movement ${ }^{1}$ ${ }^{2}$ out of Wuhan alone $(\mathrm{R} 2=0.89)$ from a log-linear regression using cumulative cases. ${ }^{2}$ ${ }^{3}$ The authors showed that although travel restrictions may have reduced the flow of ${ }^{3}$ ${ }^{4}$ case importations from Wuhan, other local mitigation strategies aimed at halting ${ }^{4}$ ${ }^{5}$ local transmission reduced the further spread of the virus. 5

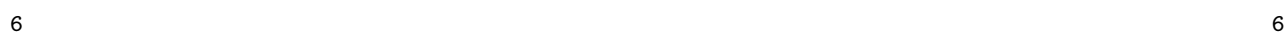

8 In a study evaluating the effect of mobility restriction in limiting COVID-19 8 ${ }_{9}$ spread, using zip code data for Atlanta, Boston, Chicago, New York (NYC), and ${ }_{9}$ ${ }_{10}$ Philadelphia, the authors estimated that total COVID-19 cases per capita decreased ${ }_{10}$ ${ }_{11}$ on average by approximately 20 percent for every ten percentage points fall in mobil- ${ }_{11}$ ${ }_{12}$ ity between February and May 2020 [17]. In another study, the correlation between ${ }_{12}$ ${ }_{13}$ the COVID-19 growth rate and travel distance decay rate and dwell time at home ${ }_{13}$ ${ }_{14}$ change rate was -0.586 (95\% CI: $\left.-0.742 \quad-0.370\right)$ and 0.526 (95\% CI: $\left.0.293 \quad 0.700\right)_{14}$ ${ }_{15}$ respectively. Increases in state-specific doubling time of total cases ranged from $_{15}$ ${ }_{16} 6.86$ days to 30.29 days after social distancing orders were put in place [4]. Another ${ }_{16}$ ${ }_{17}$ analysis across counties in the US, showed that adoption of government-imposed ${ }_{17}$ ${ }_{18}$ Social distancing measures reduced the daily growth rate of confirmed COVID-19 ${ }_{18}$ ${ }_{19}$ cases by 5.4 percentage points after one to five days, 6.8 percentage points after $\operatorname{six}_{19}$

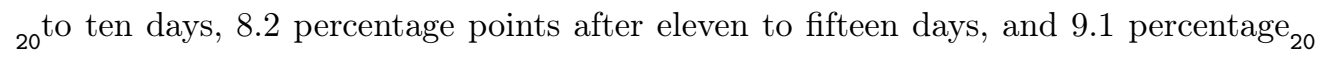
${ }_{21}$ points after sixteen to twenty days [13]. In a recent European study, it was $\operatorname{shown}_{21}$ ${ }_{22}$ that internal mobility is more important than mobility across provinces to $\operatorname{control}_{22}$ ${ }_{23}$ COVID-19, and the typical lagged positive effect of reduced human mobility $\mathrm{on}_{23}$ ${ }_{24}$ reduced human mobility on reducing excess deaths is around 14-20 days [18]. $\quad 24$

${ }^{26}$ A big question that policy makers at state, or country level jurisdictions face is ${ }^{26}$ ${ }^{27}$ if the impact of visitor mobility is different than local mobility. In this study, we ${ }^{27}$ ${ }^{28}$ examine the transmission risk propagated as a result of local mobility vs. risk prop- ${ }^{28}$ ${ }^{29}$ agated from visitor mobility for all the states in United States. We use two different ${ }^{29}$

30 . 30 variables to capture the infection propagation risk, namely the local transmission ${ }^{31}$ risk (due to local mobility) and the visitor transmission risk (due to visitor mo${ }^{32}$ bility), and evaluate the impact of these variables on case growth. This study was ${ }^{32}$ ${ }^{33}$ done across all the 50 states in United States from March 2020 to December $2020 .^{33}$ 


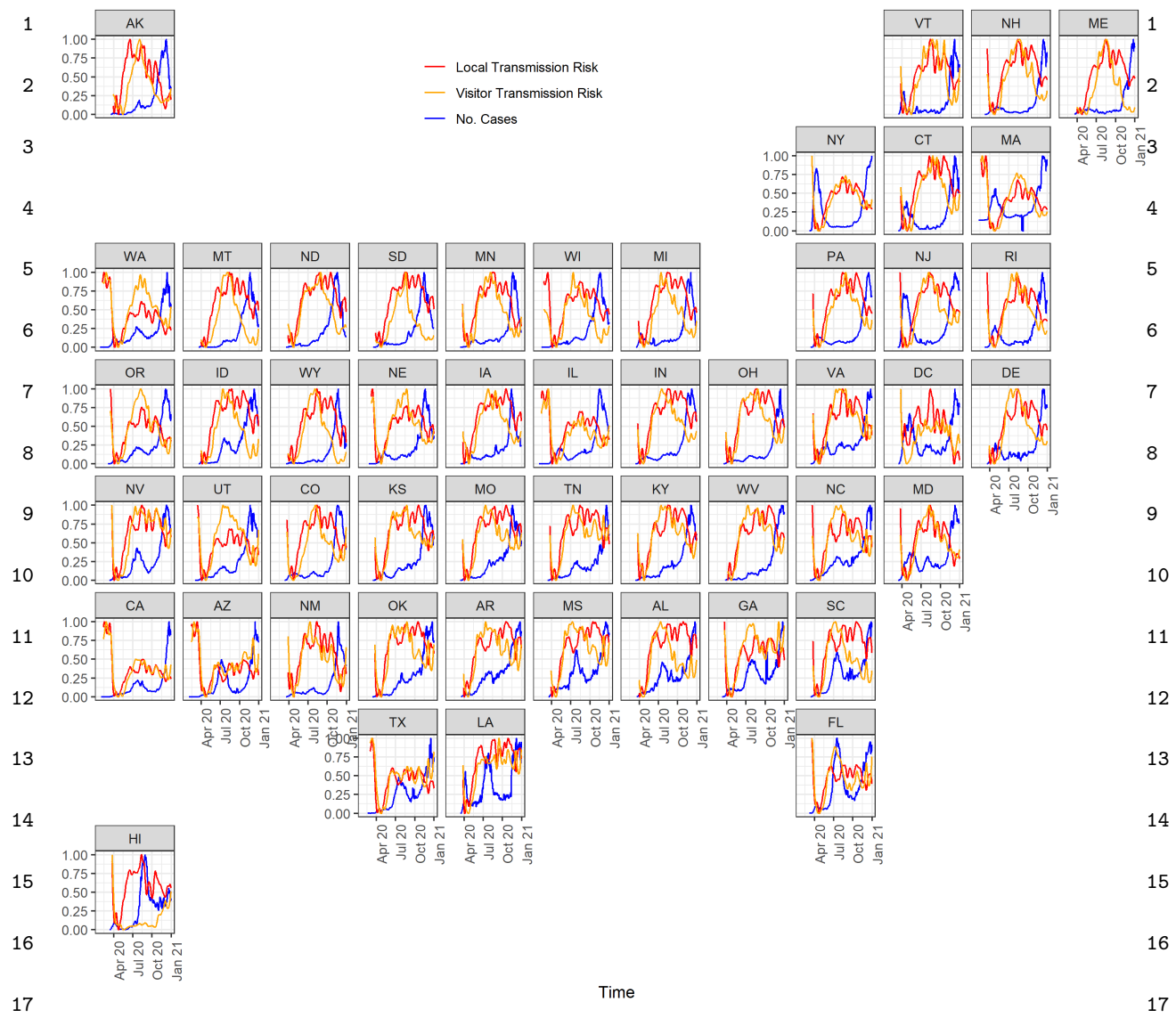

Figure 1: The timeseries of the cases per capita, local transmission risk, and visitor 18 19 transmission risk for various states in the United States

2 Methods

\section{Methods}

${ }_{24}$ The confirmed cases data was retrieved from the Corona Data Scraper open-source ${ }_{24}$ ${ }_{25}$ project [26], which provides county-level data on the number of new cases per day. ${ }_{26}$ We aggregated the number of cases to a state-level.

26

${ }^{27}$ 2.1.2 Mobility Data

${ }^{28}$ State-level mobility dataset and metrics were provided by SafeGraph [27]. Safe- ${ }^{28}$ ${ }^{29}$ Graph provides agregated trip information at a census tract level obtained from ${ }^{29}$ 30 nonymized mobile device locations. The intra-state (or local) trips represent the 30 31 trips taken by individuals starting and ending within the same state (i.e., state ${ }^{31}$ ${ }^{32}$ boundary). The inter-state (or visitor) trips are those where the origin and desti- ${ }^{32}$ ${ }^{33}$ nation are in different states (i.e., origin in one state and destination in a different ${ }^{33}$ 
${ }^{1}$ state). This data was collected for all the trips made between January 1, 2020 and $^{1}$

${ }^{2}$ December 31, 2020.

2

3

3

42.2 Approach

${ }_{5}$ In order to measure the impact of mobility (both local and visitor), we model the ${ }_{5}$ ${ }_{6}$ number of cases at a particular location based on the historical number of cases, 6 ${ }_{7}$ the transmission of infection based on the mobility. Higher accuracy when a factor ${ }_{7}$ sis included in the model shows that the particular factor is important [28]. The ${ }_{9}$ features used to forecast the number of cases is listed below: 9

10

2.2.1 Number of Cases

10

11

The aggregated new cases from the previous 14 days is used to forecast the number 12

of cases for the next 14 days; earlier studies have shown that the virus incubation

period is about 14 days [29].

14

${ }^{15}$ 2.2.2 Local transmission risk

${ }^{16}$ The local transmission risk represents the transmission potential of the virus based ${ }^{16}$

${ }^{17}$ on the recent number of cases per capita (which represents local case incidence) ${ }^{17}$

${ }^{18}$ and the mobility both at the local level. The local transmission coefficient $L T$ for $\mathrm{a}^{18}$

${ }^{19}$ spatial region $i$ is calculated using the formula: 19

20

20

21

$22 L T_{i}=M_{i, i} \times C_{i}$

21

23

22

Where $M_{i, i}$ represents the number of trips where the origin and destination of the

trips fall within the region $i$. The cases per 100,000 people at the location $i$, which

25 we denote as $C_{i}$.

26

${ }^{27}$ 2.2.3 Visitor transmission risk

26

${ }^{28}$ The visitor transmission risk represents the transmission potential of the virus based ${ }^{28}$

${ }^{29}$ on the recent number of cases per capita at the visitor origin. The visitor transmis- ${ }^{29}$

${ }^{30}$ sion $V T$ at a location $i$ can be calculated using.

31

31

32

32

33

$V T_{i}=\sum_{j=0}^{n} M_{j, i} \times C_{j}$ 
${ }^{1}$ Where $M_{j, i}$ represents the number of trips that originate at $j$ and end at location ${ }^{1}$

${ }^{2} i$ and $j \neq i$. The cases per capita at location $j$ is represented by $C_{j}$. These three ${ }^{2}$

${ }^{3}$ measures are illustrated in Figure 1.

4

${ }_{5} 2.3$ Machine Learning Methods

5

${ }_{6}$ We employ various machine learning techniques to forecast number of cases based 6 ${ }_{7}$ the local vs visitor mobility. The general idea is to evaluate if including visitor ${ }_{7}$ stransmission risk improves the forecasting performance by analyzing the relation-॰ ${ }_{9}$ ship between future number of cases and historical local mobility vs. future number ${ }_{9}$ ${ }_{10}$ of cases with visitor mobility using machine learning methods. Machine learning ${ }_{10}$ ${ }_{11}$ models are more capable of capturing the non-linear relationships between various ${ }_{11}$ ${ }_{12}$ features. The abundance of the COVID data - case data and mobility patterns, ${ }_{12}$ ${ }_{13}$ enable us to identify complex relationship patterns. In this study, three popular ${ }_{13}$ ${ }_{14}$ machine learning methods were used, namely, Linear regression, Random Forest ${ }_{14}$ ${ }_{15}$ Regression, and XGBoost Regression to forecast the number of cases. These models ${ }_{15}$ ${ }_{16}$ take into account the historical number of cases, local transmission risk and visitor ${ }_{16}$ ${ }_{17}$ transmission risk into account when forecasting the future number of cases. $\quad{ }_{17}$ The predictive performance of the proposed approach for each of the stations is 20 compared using the following two metrics: Mean absolute percentage error (MAPE) 21 measures the average percent of absolute deviation between actual and forecasted 22 values.

23

24

25

$$
M A P E=\frac{1}{N} \sum \frac{|A-P|}{A} \times 100
$$

${ }_{26}$ Root mean squared error (RMSE) captures the square root of average of squares of 26 $\begin{array}{ll}27 \text { the difference between actual and forecasted values. } & 27\end{array}$

${ }^{31}$ Where, $N$ is the number of test samples, $A$ is the actual value, and $P$ is its respective ${ }^{31}$

${ }^{32}$ predicted value. For each of the techniques, we evaluate the accuracy of prediction ${ }^{32}$

33 with and without using the visitor transmission risk with and without using the visitor transmission risk. 


\begin{tabular}{|c|c|c|c|c|}
\hline Model & No Mobility & $\begin{array}{c}\text { Local Transmission } \\
\text { Risk Only }\end{array}$ & $\begin{array}{c}\text { Visitor Transmission } \\
\text { Risk Only } \\
\end{array}$ & $\begin{array}{c}\text { Local and Visitor } \\
\text { Risk }\end{array}$ \\
\hline Gradient Boost & 0.244 & 0.222 & 0.17 & 0.168 \\
\hline Linear Regression & 0.512 & 0.464 & 0.536 & 0.497 \\
\hline Random Forest & 0.335 & 0.327 & 0.309 & 0.306 \\
\hline
\end{tabular}

${ }^{4}$ Table 1: Comparison of MAPE to forecast the number of cases with and without ${ }^{4}$

5 visitor transmission risk for various machine learning models

6

\begin{tabular}{|c|c|c|c|c|}
\hline Model & No Mobility & $\begin{array}{l}\text { Local Transmission } \\
\text { Risk Only }\end{array}$ & $\begin{array}{l}\text { Visitor Transmission } \\
\text { Risk Only }\end{array}$ & $\begin{array}{l}\text { Local and Visitor } \\
\text { Transmission Risk }\end{array}$ \\
\hline Gradient Boost & 0.084 & 0.075 & 0.055 & 0.055 \\
\hline Linear Regression & 0.108 & 0.105 & 0.102 & 0.099 \\
\hline Random Forest & 0.075 & 0.074 & 0.063 & 0.063 \\
\hline
\end{tabular}

${ }^{10}$ Table 2: Comparison of RMSE to forecast the number of cases with and without ${ }^{10}$

${ }^{11}$ visitor transmission risk for various machine learning models 11

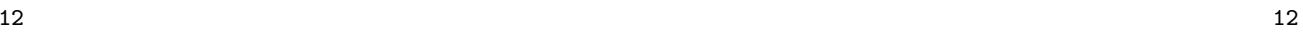

13 Diebold-Mariano test (DM-test) is used to evaluate the significance of the pre-

14 dictions of the two models [30]. The models use the forecasted number of cases 15 generated with and without using the visitor transmission for each of the three 16 machine learning approaches. The null hypothesis of the DM-test is that the two 17 forecasts have similar forecast accuracy. The alternative or rejection of the null hy18 pothesis is that the two forecasts have significantly different forecasting accuracy, 19 i.e., the forecasts are not similar using the two models.

20

${ }^{21} 3$ Results 21

${ }^{22}$ Tables 1 and 2 show the comparison of the machine learning forecasts with and ${ }^{22}$ ${ }^{23}$ without the inclusion of visitor mobility and local mobility. We compare the perfor- ${ }^{23}$ ${ }^{24}$ mance of the three machine learning models (Gradient Boost, Linear Regression ${ }^{24}$ ${ }^{25}$ and Random Forecast) using the MAPE and RMSE. In addition a DM test was ${ }^{25}$ ${ }^{26}$ performed to evaluate the significance of forecasts when visitor mobility is included ${ }^{26}$ ${ }^{27}$ in the model.

28 Table 1 shows the MAPE of machine learning models for the complete duration ${ }^{28}$ ${ }^{29}$ (i.e. March 2020 to December 2020). We observe that the MAPE of the best per- ${ }^{29}$ ${ }^{30}$ forming model (i.e. Gradient Boost) has a 30 forming model (i.e. Gradient Boost) has a MAPE of $16.8 \%$ when using visitor mo${ }^{31}$ bility compared to $22 \%$ when just the local transmission risk is taken into account. ${ }^{32}$ Linear regression model shows better performance when using local transmission ${ }^{32}$ ${ }^{33}$ risk than combined local and visitor transmission risk. However, Table 2 shows that ${ }^{33}$ 


\begin{tabular}{|c|c|c|c|c|}
\hline Model & No Mobility & $\begin{array}{c}\text { Local Transmission } \\
\text { Risk Only }\end{array}$ & $\begin{array}{c}\text { Visitor Transmission } \\
\text { Risk Only } \\
\end{array}$ & $\begin{array}{l}\text { Local and Visitor } \\
\text { Transmission Risk }\end{array}$ \\
\hline \multicolumn{5}{|c|}{ First Wave (March - June) } \\
\hline Gradient Boost & 0.376 & 0.308 & 0.169 & 0.174 \\
\hline Linear Regression & 1.064 & 1.029 & 1.094 & 0.938 \\
\hline Random Forest & 0.422 & 0.409 & 0.329 & 0.327 \\
\hline \multicolumn{5}{|c|}{ Second Wave (July - September) } \\
\hline Gradient Boost & 1.038 & 0.999 & 0.885 & 0.834 \\
\hline Linear Regression & 1.411 & 1.35 & 1.251 & 1.208 \\
\hline Random Forest & 1.023 & 1.007 & 0.887 & 0.875 \\
\hline \multicolumn{5}{|c|}{ Third Wave (October - December) } \\
\hline Gradient Boost & 0.469 & 0.492 & 0.352 & 0.341 \\
\hline Linear Regression & 1.401 & 1.369 & 1.174 & 1.142 \\
\hline Random Forest & 0.498 & 0.508 & 0.369 & 0.359 \\
\hline
\end{tabular}

${ }_{10}$ Table 3: Comparison of forecasting performance using MAPE with and without ${ }_{10}$ ${ }_{11}$ local and visitor transmission risk for three waves using various machine learning $_{11}$ ${ }_{12}$ models ${ }_{15}$ the RMSE is lower when using the combination of local and visitor transmission ${ }_{15}$ ${ }_{16}$ risk than local transmission risk. In addition, the RMSE is lower when local and ${ }_{16}$ ${ }_{17}$ visitor mobility is used for all the three models.

18 The significance of the results is evaluated using the Diebold-Mariano score that 18 19evaluates the null hypothesis that both the forecasts are the same, the p-value shows19 20that the null hypothesis is rejected and the difference in forecasts are statistically20 21significant. The DM scores for the gradient boost, linear regression, and random21 22forest are $8.7(\mathrm{p}=0.2), 2.66(\mathrm{p}=0.04)$, and $8.26(\mathrm{p}=0.04)$ respectively. The MAPE and22 23RMSE on the state level data in Table 5 shows that the inclusion of external mobility23 24leads to better forecasts for all 50 states in the United States. 24 ${ }^{25}$ We make similar observations when the data is separated into three waves (Tables ${ }^{25}$ ${ }^{26} 3$ and 4 ). The MAPE and RMSE for all the three approaches report lower MAPE ${ }^{26}$ ${ }^{27}$ and RMSE when visitor transmission risk is included in the model generation com- ${ }^{27}$ ${ }^{28}$ pared to when local mobility is used. The inclusion of the visitor transmission risk ${ }^{28}$ ${ }^{29}$ improves the MAPE of the forecast by about $57.19 \%$ on average across all states for ${ }^{29}$ 30 the three waves. During the first phase, the external mobility results in a decrease in ${ }^{30}$ ${ }^{31}$ MAPE by $110 \%$ with the percentage error at $17.14 \pm 0.28 \%$ and $30.81 \pm 0.48 \%$ with $^{31}$ ${ }^{32}$ external and only local transmission coefficient respectively. Similarly, the improve- ${ }^{32}$ 33 . 33 ment for MAPE for the phase 2 and 3 are $34.23 \%$ and $26.17 \%$ respectively. 


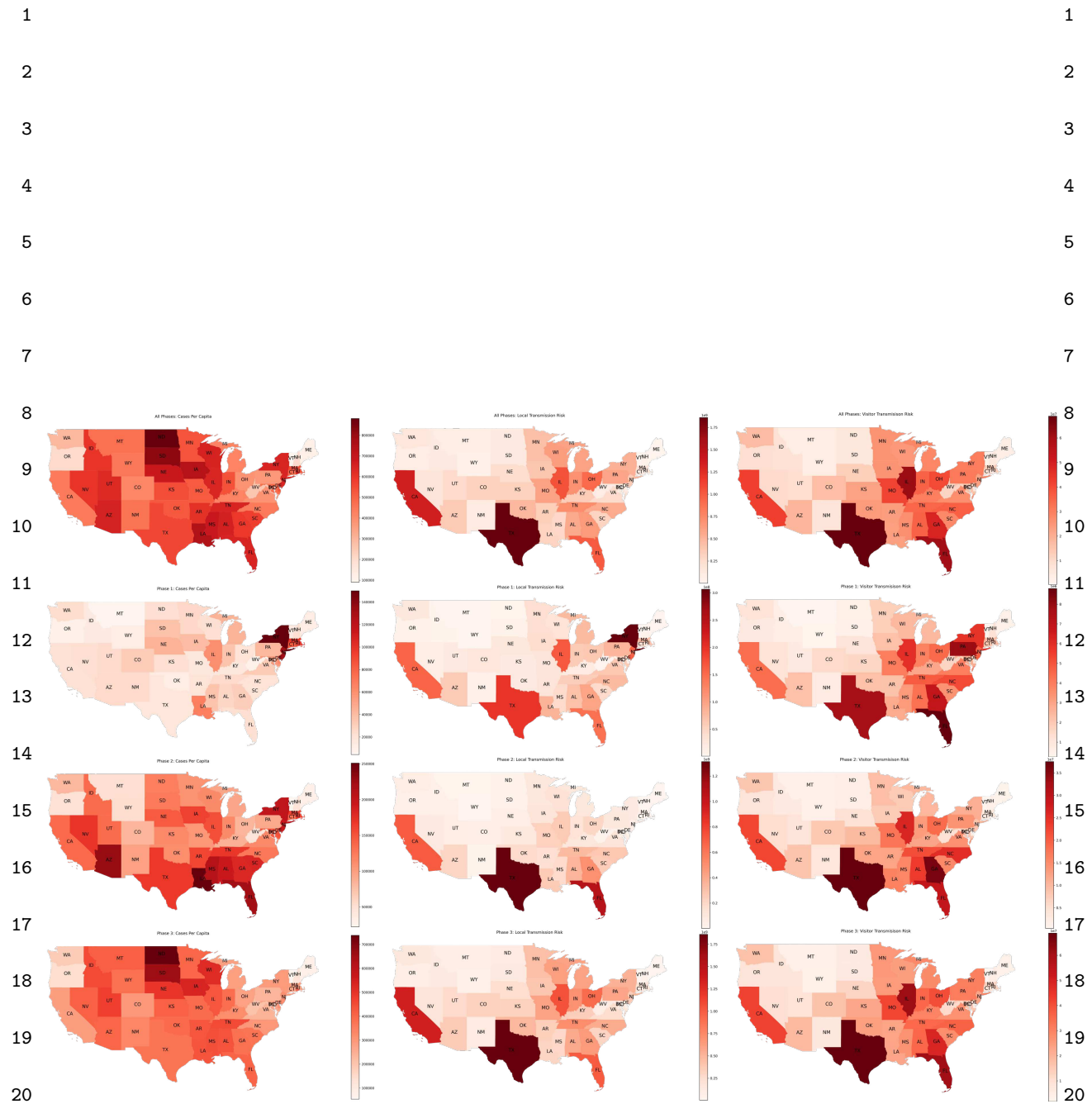

20

Figure 2: Distribution of the number of cases, Local Transmission Risk and Visitor ${ }_{21}$ ${ }_{22}$ Transmission Risk to various states across all phases (March - December), phase ${ }_{22}$ ${ }_{23}^{1}$ (March - June), phase 2 (July - August), and phase 3 (October - December) ${ }_{23}$ ${ }_{24}$ Certain states that have high number of cases have high local transmission risk, ${ }_{24}$ ${ }_{25}$ where as others have high visitor transmission risk, where risk is imported from ${ }_{25}$ ${ }_{26}{ }^{\text {outside the state boundaries. }}$ 
${ }^{1} 4$ Discussion of Results

${ }^{2}$ Figure 2 shows the cumulative number of cases per capita, local transmission risk, ${ }^{2}$

${ }^{3}$ and the visitor transmission risk for each of the states in the United States for $^{3}$

${ }^{4}$ the entire pandemic and the three phases of the pandemic. We observe that certain ${ }^{4}$

${ }^{5}$ states have a lower local transmission risk and a higher visitor transmission risk. For ${ }^{5}$

${ }^{6}$ example, during the second phase of the pandemic, states like Illinois and Georgia, ${ }^{6}$

${ }^{7}$ the local transmission risk is much lower than the transmission risk posed by trav- $^{7}$

${ }^{8}$ elers from other states to Illinois. Similarly, for states like New York in phase 1, the ${ }^{8}$

${ }^{9}$ local transmission risk is higher than the visitor transmission risk. There are also ${ }^{9}$

${ }^{10}$ variations between the interplay of local and visitor transmission risks for different ${ }^{10}$

${ }^{11}$ phases of the pandemic. The first phase is primarily driven by local mobility, and ${ }^{11}$

${ }^{12}$ the other two phases are a combination of local and visitor mobility. 12

13
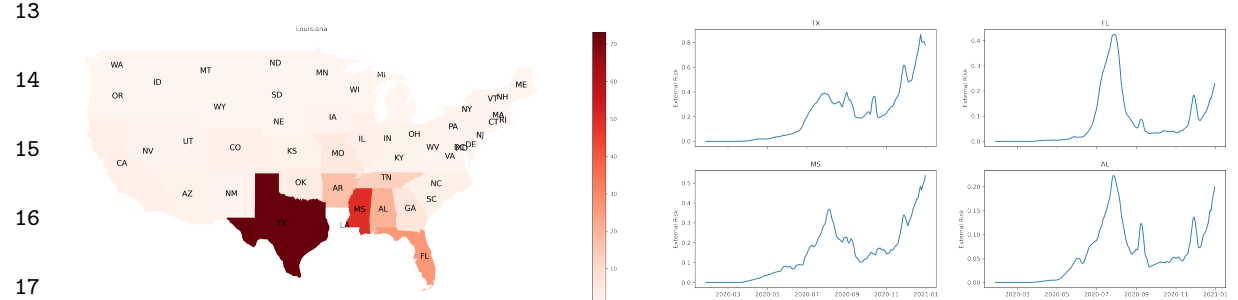

13

14

15

16

18 (a) Distribution of visitors to Louisiana

(b) Time series of visitor transmission 18

19 from other states in the U.S.

risk to Louisiana from neighboring states

19

20Figure 3: Visitor transmission risk and mobility patterns to the state of Louisiana2o 21from other states in the United States

(a) Distribution of visitors to New York from other states in the U.S.
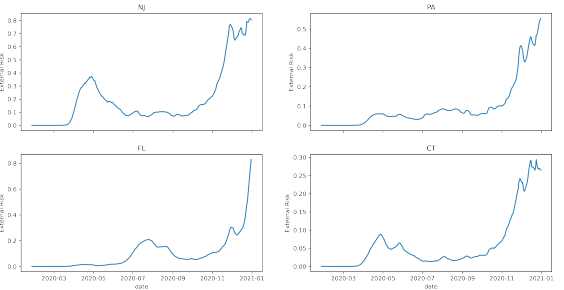

(b) Time series of visitor transmission risk to New York from neighboring states
22

23

24

25

26

27

28

Figure 4: Visitor transmission risk and mobility patterns to the state of New York 30 from other states in the United States

31

${ }^{32}$ While it is apparent that the majority of the visitor transmission risk is due to ${ }^{32}$ ${ }^{33}$ travelers crossing state boundaries from neighboring cities, there is also consider- ${ }^{33}$ 


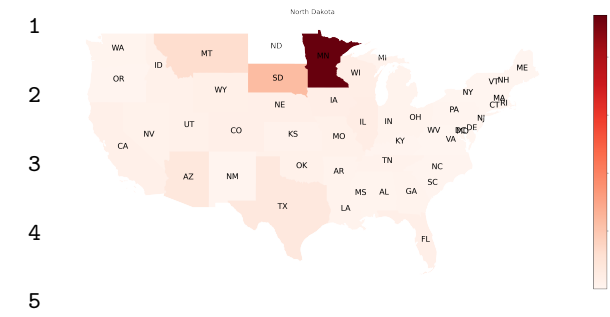

(a) Distribution of visitors to North

${ }^{6}$ Dakota from other states in the U.S.
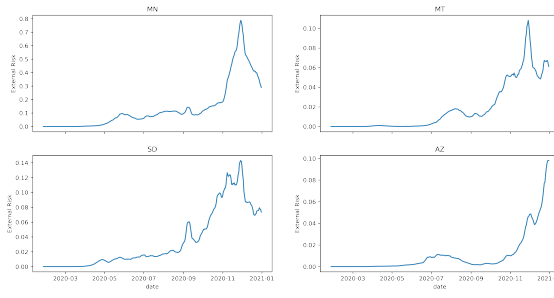

(b) Time series of visitor transmission risk to North Dakota from neighboring states
2

5

7

${ }^{8}$ Figure 5: Visitor transmission risk and mobility patterns to the state of North $^{8}$

${ }^{9}$ Dakota from other states in the United States

10

${ }^{11}$ able transmission risk due to long-distance travel. For example, for Louisiana, the ${ }^{11}$

12 in of the risk for its second peak is contributed by Mississippi, Texas, and ${ }^{12}$

33

Florida, which is higher than the Arkansas that borders the state in the north.

${ }^{14}$ The states of Mississippi and Florida contributed more to the second peak, whereas ${ }^{14}$

${ }^{15}$ travelers from Texas contributed to the second and third phases of the pandemic. ${ }^{15}$

${ }^{16}$ For the states like North Dakota, most of the visitor transmission risk is attributed ${ }^{16}$

17

to their neighboring states. New York, on the other hand, has a huge increase

${ }^{18}$ in visitor transmission risk from Florida during the late winter when the state of ${ }^{18}$

${ }^{19}$ Florida opened up to travelers compared to the rest of the country. These trends ${ }^{19}$

20

are presented in Figures 3, 4 and 5

21

${ }^{22} 5$ Limitations and Future Work 22

${ }^{23}$ This study presents a novel approach to examine the association between the effect ${ }^{23}$

${ }^{24}$ of local and visitor mobility on the number of cases for various states in the United ${ }^{24}$

${ }^{25}$ States. There are several areas where this study can be potentially improved. First, ${ }^{25}$

${ }^{26}$ this study focuses on the association between the internal and external mobility and ${ }^{26}$

${ }^{27}$ their effect on the increases in the number of cases. This study does not consider the ${ }^{27}$

$28 \quad 28$ many other factors like usage of masks, social distancing, the impact of regulations,

${ }^{29}$ and the varying compliance from the public that could have had an effect on the ${ }^{29}$

${ }^{30}$ number of cases. Second, the associations are computed at a daily granularity. The

${ }^{31}$ majority of the states have issues related to non-uniform cases where a higher num- ${ }^{31}$

${ }^{32}$ ber of cases are reported over Mondays and Tuesdays. In comparison, the number ${ }^{32}$

${ }^{33}$ of cases reported over the weekend is lower. We solved this problem by smoothening ${ }^{33}$ 
${ }^{1}$ the data over seven days. Third, the case data might be prone to errors due to both ${ }^{1}$

${ }^{2}$ reporting issues and the outliers in testing when states update their case numbers ${ }^{2}$ ${ }^{3}$ post-hoc. Fourth, the mobility data considers the number of individuals traveling ${ }^{3}$ ${ }^{4}$ from one state to another, but does not capture the distance traveled by individ- ${ }^{4}$ ${ }^{5}$ uals during the trip. Incorporating the distance traveled might help enhance the ${ }^{5}$ ${ }^{6}$ relationship between the number of cases and the mobility of individuals. Finally, ${ }^{6}$ ${ }^{7}$ we consider the state as a single unit to measure the mobility and the number of ${ }^{7}$ ${ }^{8}$ cases. We do not consider the population density at origin and destination and the ${ }^{8}$ ${ }^{9}$ number of people traveling to a particular city in a state. For example, the first ${ }^{9}$ ${ }^{10}$ wave (March - June) was dominated by cases from metropolitan areas, whereas the ${ }^{10}$ ${ }^{11}$ cases during the third wave were primarily in the rural areas of the state. In the ${ }^{11}$ ${ }^{12}$ future, we would like to extend this model for various metropolitan areas in the ${ }^{12}$ ${ }^{13}$ county for analysis at a more refined level of granularity. ${ }^{13}$

16In this paper, we evaluated the impact of the disease transmission risk due to16 ${ }^{17}$ visitor and local mobility on the number of cases at a state level for all 50 states17 18in the United States. We observed that visitor mobility is an important factor18 19to explain case growth. The prediction accuracy improved by $29 \%$ for the whole19 20duration of the pandemic in 2020 (March - December) when visitor mobility was 20 ${ }^{21}$ used in the forecasting model. The impact of transmission risk due to external21 22mobility is observed across all three phases of the pandemic in the United States. We22 23observe the influence of mobility is much stronger in the first phase of the pandemic23 ${ }^{24}$ compared to the second or third phase. These observations are consistent with24 ${ }^{25}$ some of the earlier studies $[4,11]$ where mobility was observed to be an important 25 26predictor for case growth in the first phase of the pandemic. 26 27 27

${ }_{28}$ Appendix 28 
Conceptualization: R.G.; Methodology: S.K.; Software: S.K.; Validation: S.K. and R.G.; Formal analysis: R.B.

4 and R.G.; Investigation: R.B. and Z.A.; Resources: R.G.; Data curation: R.B. and S.K.; Writing一original draft 4 ${ }_{5}$ preparation: R.G. and R.B.; Writing-review and editing: Z.A. and V.R.; Visualization: R.B.; Supervision: R.G.; 5 Project administration: R.G.; Funding acquisition: R.G. All authors reviewed the manuscript.

6

Acknowledgements

7 None

8 Availability of data and materials

The analysis code for this paper is available on GitHub at

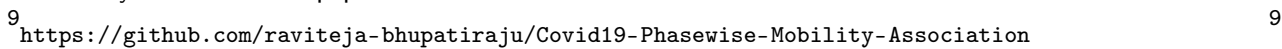

${ }^{10}$ Author details 10

11 Informatics Research Institute, University of Louisiana at Lafayette, Lafayette, USA. 11

${ }_{12}$ References 12

1. Grantz, K.H., Meredith, H.R., Cummings, D.A., Metcalf, C.J.E., Grenfell, B.T., Giles, J.R., Mehta, S.,

13 Solomon, S., Labrique, A., Kishore, N., et al.: The use of mobile phone data to inform analysis of covid-1913 pandemic epidemiology. Nature communications 11(1), 1-8 (2020)

2. Buckee, C.O., Balsari, S., Chan, J., Crosas, M., Dominici, F., Gasser, U., Grad, Y.H., Grenfell, B.,

15 Halloran, M.E., Kraemer, M.U., et al.: Aggregated mobility data could help fight covid-19. Science (New 15 York, NY) 368(6487), 145 (2020). doi:10.1126/science.abb8021

16 3. Franch-Pardo, I., Napoletano, B.M., Rosete-Verges, F., Billa, L.: Spatial analysis and gis in the study of 16

17 covid-19. a review. Science of The Total Environment, 140033 (2020). 17 doi:10.1016/j.scitotenv.2020.140033

18 4. Gao, S., Rao, J., Kang, Y., Liang, Y., Kruse, J.: Mapping county-level mobility pattern changes in the 18

19 united states in response to covid-19. SIGSPATIAL Special 12(1), 16-26 (2020). 19 doi:10.1145/3404820.3404824"

20 5. Oliver, N., Lepri, B., Sterly, H., Lambiotte, R., Deletaille, S., De Nadai, M., Letouzé, E., Salah, A.A., 20

21 Benjamins, R., Cattuto, C., et al.: Mobile phone data for informing public health actions across the 21 COVID-19 pandemic life cycle. American Association for the Advancement of Science (2020).

22 doi:10.1126/sciadv.abc0764

6. Warren, M.S., Skillman, S.W.: Mobility changes in response to COVID-19. Preprint at

23 https://arxiv.org/abs/2003.14228 (2020)

24 7. Queiroz, L., Ferraz, A., Melo, J.L., Barboza, G., Urbanski, A.H., Nicolau, A., Oliva, S., Nakaya, H.: 24 Large-scale assessment of human mobility during covid-19 outbreak (2020)

25 8. Aktay, A., Bavadekar, S., Cossoul, G., Davis, J., Desfontaines, D., Fabrikant, A., Gabrilovich, E., Gadepalli, 25

26 K., Gipson, B., Guevara, M., et al.: Google COVID-19 community mobility reports: Anonymization process 26 description (version 1.0). Preprint at https://arxiv.org/abs/2004.04145 (2020)

27 9. Hartley, D.M., Perencevich, E.N.: Public health interventions for covid-19: emerging evidence and 27 implications for an evolving public health crisis. Jama 323(19), 1908-1909 (2020).

28 doi:10.1001/jama.2020.5910

2910. Kuchler, T., Russel, D., Stroebel, J.: The geographic spread of covid-19 correlates with structure of social 29 networks as measured by facebook. Technical report, National Bureau of Economic Research (2020)

${ }^{30} 11$. Badr, H.S., Du, H., Marshall, M., Dong, E., Squire, M.M., Gardner, L.M.: Association between mobility 30

31 patterns and covid-19 transmission in the usa: a mathematical modelling study. The Lancet Infectious 31 Diseases 20(11), 1247-1254 (2020). doi:10.1016/S1473-3099(20)30553-3"

3212. Basellini, U., Alburez-Gutierrez, D., Del Fava, E., Perrotta, D., Bonetti, M., Camarda, C.G., Zagheni, E.: 32 33 Linking excess mortality to Google mobility data during the COVID-19 pandemic in England and Wales. 33 Preprint at https://osf.io/preprints/socarxiv/75d6m/ (2020) 
13. Courtemanche, C., Garuccio, J., Le, A., Pinkston, J., Yelowitz, A.: Strong social distancing measures in the ${ }^{1}$

2 united states reduced the covid-19 growth rate: Study evaluates the impact of social distancing measures 2 on the growth rate of confirmed covid-19 cases across the united states. Health Affairs 39(7), 1237-1246

3 (2020)

44. Engle, S., Stromme, J., Zhou, A.: Staying at home: mobility effects of covid-19. Available at https://ssrn. com/abstract=3565703 (2020)

515. Gao, S., Rao, J., Kang, Y., Liang, Y., Kruse, J., Dopfer, D., Sethi, A.K., Reyes, J.F.M., Yandell, B.S., 5 Patz, J.A.: Association of mobile phone location data indications of travel and stay-at-home mandates with covid-19 infection rates in the us. JAMA network open 3(9), 2020485-2020485 (2020).

7 doi:10.1001/jamanetworkopen.2020.20485

Y., Kruse, J., Doepfer, D., Sethi, A.K., Reyes, J.F.M., Patz, J., Yandell,

B.S.: Mobile phone location data reveal the effect and geographic variation of social distancing on the 8

spread of the COVID-19 epidemic. Preprint at https://arxiv.org/abs/2004.11430 (2020)

17. Glaeser, E.L., Gorback, C.S., Redding, S.J.: How much does covid-19 increase with mobility? evidence

10 from new york and four other us cities. Technical report, National Bureau of Economic Research (2020). 10 Available at https://doi.org/10.3386/w27519

11

18. lacus, S.M., Santamaria, C., Sermi, F., Spyratos, S., Tarchi, D., Vespe, M.: Human mobility and covid-19

12 initial dynamics. Nonlinear Dynamics 101(3), 1901-1919 (2020) 12

19. Linka, K., Peirlinck, M., Sahli Costabal, F., Kuhl, E.: Outbreak dynamics of covid-19 in europe and the

13 effect of travel restrictions. Computer Methods in Biomechanics and Biomedical Engineering 23(11) $710-717(2020)$

20. Vinceti, M., Filippini, T., Rothman, K.J., Ferrari, F., Goffi, A., Maffeis, G., Orsini, N.: Lockdown timing

15 and efficacy in controlling covid-19 using mobile phone tracking. EClinicalMedicine 25, 100457 (2020).

16 doi:10.1016/j.eclinm.2020.100457

21. Beria, P., Lunkar, V.: Presence and mobility of the population during the first wave of covid-19 outbreak

17 and lockdown in italy. Sustainable Cities and Society 65, 102616 (2021)

22. Liu, A., Vici, L., Ramos, V., Giannoni, S., Blake, A.: Visitor arrivals forecasts amid covid-19: A perspective ${ }_{18}$ from the europe team. Annals of Tourism Research 88, 103182 (2021)

1923. Qiu, R.T., Wu, D.C., Dropsy, V., Petit, S., Pratt, S., Ohe, Y.: Visitor arrivals forecasts amid covid-19: A 19 perspective from the asia and pacific team. Annals of Tourism Research 88, 103155 (2021)

${ }^{20} 24$. Yamamoto, M.: Analyzing the impact of the spread of covid-19 infections on people's movement in tourist 20

21 destinations. Journal of Global Tourism Research 6(1), 37-44 (2021) 21

25. Kraemer, M.U., Yang, C.-H., Gutierrez, B., Wu, C.-H., Klein, B., Pigott, D.M., Du Plessis, L., Faria, N.R.,

$22 \mathrm{Li}, \mathrm{R}$. , Hanage, W.P., et al.: The effect of human mobility and control measures on the covid-19 epidemic 22

23 in china. Science 368(6490), 493-497 (2020)

26. Larry Davis: Corona Data Scrapper. https://coronadatascraper.com/. (Date of Access: 2021-06-30)

27. SafeGraph: Safegraph Data Consortium. https://www.safegraph.com/covid-19-data-consortium. (Date of Access: 2021-06-30) (2020)

2628. Bolón-Canedo, V., Alonso-Betanzos, A.: Ensembles for feature selection: A review and future trends. 26 Information Fusion 52, 1-12 (2019)

${ }^{27} 29$. Lei, S., Jiang, F., Su, W., Chen, C., Chen, J., Mei, W., Zhan, L.-Y., Jia, Y., Zhang, L., Liu, D., et al.: 27

28 Clinical characteristics and outcomes of patients undergoing surgeries during the incubation period of 28 covid-19 infection. EClinicalMedicine 21, 100331 (2020)

2930. Diebold, F.X., Mariano, R.S.: Comparing predictive accuracy. Journal of Business \& economic statistics 29

$30 \quad 20(1), 134-144(2002)$ 


\begin{tabular}{|c|c|c|c|c|c|c|c|c|c|c|c|c|c|c|c|c|}
\hline \multirow{3}{*}{ FIPS } & \multicolumn{4}{|c|}{ All Waves } & \multicolumn{4}{|c|}{ First Wave } & \multicolumn{4}{|c|}{ Second Wave } & \multicolumn{4}{|c|}{ Third Wave } \\
\hline & \multicolumn{2}{|l|}{ MAPE } & \multicolumn{2}{|l|}{ RMSE } & \multicolumn{2}{|l|}{ MAPE } & \multicolumn{2}{|l|}{ RMSE } & \multicolumn{2}{|l|}{ MAPE } & \multicolumn{2}{|l|}{ RMSE } & \multicolumn{2}{|l|}{ MAPE } & \multicolumn{2}{|l|}{ RMSE } \\
\hline & Visitor + Local & Local & Visitor + Local & Local & Visitor + Local & Local & Visitor + Local & Local & Visitor + Local & Local & Visitor + Local & \begin{tabular}{|l|} 
Local \\
\end{tabular} & Visitor + Local & Local & Visitor + Local & Local \\
\hline 1 & 0.39 & 0.43 & 0.13 & 0.14 & 0.31 & 0.53 & 0.14 & 0.18 & 1.78 & 2.79 & 0.23 & 0.36 & 0.84 & 0.51 & 0.12 & 0.12 \\
\hline 2 & 0.5 & 0.68 & 0.09 & 0.12 & 0.73 & 1.48 & 0.15 & 0.22 & 0.33 & 0.84 & 0.1 & 0.16 & 1.22 & 0.62 & 0.13 & 0.13 \\
\hline 4 & 0.79 & 1.04 & 0.15 & 0.23 & 0.33 & 0.5 & 0.11 & 0.17 & 1.54 & 2.33 & 0.17 & 0.28 & 1.06 & 1.21 & 0.09 & 0.11 \\
\hline 5 & 0.23 & 0.29 & 0.09 & 0.13 & 0.3 & 0.47 & 0.11 & 0.12 & 0.56 & 0.71 & 0.12 & 0.14 & 1.92 & 2.01 & 0.25 & 0.32 \\
\hline 6 & 0.45 & 0.74 & 0.14 & 0.2 & 0.22 & 0.21 & 0.13 & 0.12 & 3.72 & 4.09 & 0.26 & 0.33 & 1.05 & 3.15 & 0.09 & 0.21 \\
\hline 8 & 0.49 & 1.31 & 0.11 & 0.26 & 0.14 & 0.26 & 0.08 & 0.11 & 0.75 & 2.06 & 0.15 & $\begin{array}{ll}0.27 \\
\end{array}$ & 0.32 & 0.3 & 0.11 & 0.12 \\
\hline$r^{9}$ & 0.73 & 1.61 & 0.12 & 0.23 & 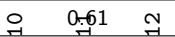 & $0 \times 92$ & 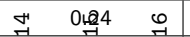 & 0,26 & 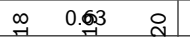 & 0.98 & $\approx 0 . \frac{d x}{\alpha}$ & 0.19 & $\stackrel{0}{0} \quad 0.2 z$ & $0.1 \mathrm{~d}$ & \& 0.0 悲 & $0.0 \mathrm{~m}$ \\
\hline 10 & 0.35 & 0.47 & 0.14 & 0.18 & 0.25 & 0.69 & 0.16 & 0.17 & 0.55 & 0.7 & 0.14 & 0.15 & 0.91 & 1.15 & 0.11 & 0.27 \\
\hline 12 & 0.52 & 0.69 & 0.16 & 0.24 & 0.44 & 0.69 & 0.13 & 0.22 & 1.46 & 1.18 & 0.21 & 0.22 & 0.5 & 2.08 & 0.1 & 0.32 \\
\hline 13 & 0.38 & 0.59 & 0.14 & 0.19 & 0.31 & 0.35 & 0.14 & 0.17 & 2 & 1.98 & 0.18 & 0.25 & 2.29 & 3.24 & 0.19 & 0.27 \\
\hline 15 & 1.04 & 1.34 & 0.09 & 0.15 & 1.68 & 2.73 & 0.2 & 0.3 & 0.33 & 1 & 0.07 & 0.19 & 0.81 & 2.18 & 0.09 & 0.23 \\
\hline 16 & 0.49 & 0.63 & 0.09 & 0.11 & 0.48 & 0.89 & 0.17 & 0.19 & 0.54 & 1.47 & 0.12 & 0.18 & 0.31 & 0.25 & 0.08 & 0.08 \\
\hline 17 & 0.46 & 0.74 & 0.12 & 0.22 & 0.24 & 0.43 & 0.12 & 0.16 & 0.44 & 0.37 & 0.14 & 0.16 & 0.17 & 0.13 & 0.05 & 0.04 \\
\hline 18 & 0.62 & 0.77 & 0.15 & 0.19 & 0.1 & 0.29 & 0.05 & 0.07 & 0.94 & 0.99 & 0.18 & 0.18 & 0.18 & 0.14 & 0.05 & 0.04 \\
\hline 19 & 0.45 & 0.49 & 0.13 & 0.14 & 0.1 & 0.33 & 0.05 & 0.07 & 0.45 & 0.44 & 0.15 & 0.16 & 0.35 & 0.27 & 0.09 & 0.09 \\
\hline 20 & 0.3 & 0.35 & 0.09 & 0.08 & 0.25 & 0.36 & 0.11 & 0.16 & 0.76 & 0.84 & 0.15 & 0.17 & 1.08 & 0.89 & 0.22 & 0.22 \\
\hline 21 & 0.34 & 0.35 & 0.07 & 0.08 & 0.11 & 0.23 & 0.04 & 0.07 & 0.45 & 0.51 & 0.1 & 0.11 & 0.52 & 0.34 & 0.1 & 0.14 \\
\hline 22 & 0.45 & 0.56 & 0.2 & 0.23 & 0.13 & 0.27 & 0.08 & 0.1 & 2.09 & 2.42 & 0.31 & 0.32 & 2.96 & 2.74 & 0.25 & 0.26 \\
\hline 23 & 0.5 & 0.6 & 0.09 & 0.12 & 0.17 & 0.8 & 0.09 & 0.16 & 0.95 & 0.87 & 0.18 & 0.21 & 0.76 & 1.21 & 0.08 & 0.09 \\
\hline 24 & 0.45 & 0.5 & 0.18 & 0.21 & 0.12 & 0.27 & 0.07 & 0.08 & 0.63 & 0.69 & 0.19 & 0.2 & 0.5 & 0.45 & 0.06 & 0.07 \\
\hline 25 & 1.14 & $\begin{array}{l}1.19 \\
\end{array}$ & 0.2 & $\begin{array}{l}0.22 \\
\end{array}$ & 0.53 & $\begin{array}{l}0.83 \\
\end{array}$ & 0.22 & 0.22 & 0.42 & $\begin{array}{l}0.46 \\
\end{array}$ & 0.19 & 0.22 & 0.44 & 0.58 & 0.05 & 0.06 \\
\hline 26 & 0.64 & 1.06 & 0.17 & 0.25 & 0.21 & 0.74 & 0.07 & 0.16 & 0.88 & 0.96 & 0.17 & 0.21 & 0.31 & 0.14 & 0.06 & 0.06 \\
\hline 27 & 0.2 & 0.64 & 0.04 & 0.16 & 0.14 & 0.24 & 0.09 & 0.08 & 0.67 & 1.05 & 0.22 & 0.22 & 0.22 & 0.16 & 0.06 & 0.05 \\
\hline 28 & 0.33 & 0.47 & 0.12 & 0.19 & 0.16 & 0.3 & 0.1 & 0.11 & 1.98 & 2.12 & 0.26 & 0.3 & 0.99 & 1.22 & 0.11 & 0.12 \\
\hline 29 & 0.55 & 0.59 & 0.1 & 0.11 & 0.16 & 0.38 & 0.08 & 0.11 & 0.78 & 0.86 & 0.1 & 0.12 & 0.98 & 1.06 & 0.23 & 0.22 \\
\hline 30 & 0.44 & 0.49 & 0.07 & 0.09 & 1.12 & 1.65 & 0.11 & 0.15 & 0.26 & 0.35 & 0.09 & 0.11 & 0.49 & 0.74 & 0.12 & 0.2 \\
\hline 31 & 0.31 & 0.34 & 0.07 & 0.08 & 0.2 & 0.39 & 0.09 & 0.12 & 1.3 & 2.24 & 0.22 & 0.25 & 0.35 & 0.37 & 0.08 & 0.09 \\
\hline 32 & 0.86 & 0.9 & 0.19 & 0.21 & 0.23 & 0.38 & 0.12 & 0.15 & 1.07 & 1.62 & 0.19 & 0.31 & 0.68 & 0.58 & 0.1 & 0.08 \\
\hline 33 & 1.48 & 1.38 & 0.21 & 0.22 & 0.14 & 0.67 & 0.07 & 0.13 & 0.63 & 0.84 & 0.15 & 0.16 & 0.74 & 0.85 & 0.11 & 0.14 \\
\hline 34 & 0.73 & 1.03 & 0.18 & 0.23 & 0.54 & 0.58 & 0.23 & 0.25 & 0.5 & 0.71 & 0.16 & 0.21 & 0.5 & 0.39 & 0.05 & 0.05 \\
\hline 35 & 0.43 & 0.51 & 0.09 & 0.11 & 0.1 & 0.41 & 0.06 & 0.08 & 1.39 & 1.97 & 0.22 & 0.29 & 0.36 & 0.34 & 0.05 & 0.05 \\
\hline 36 & 0.82 & 1.3 & 0.18 & 0.26 & 0.21 & 1.13 & 0.06 & 0.32 & 0.38 & 0.36 & 0.14 & 0.14 & 0.29 & 0.27 & 0.03 & 0.03 \\
\hline 37 & 0.23 & 0.3 & 0.08 & 0.09 & 0.27 & 0.44 & 0.14 & 0.15 & 1.17 & 1.41 & 0.19 & 0.21 & 1.44 & 1.13 & 0.2 & 0.2 \\
\hline 38 & 0.41 & 0.56 & 0.08 & 0.12 & 0.17 & 0.54 & 0.09 & 0.11 & 0.41 & 0.4 & 0.07 & 0.07 & 0.71 & 1.6 & 0.16 & 0.26 \\
\hline 39 & 0.67 & 0.95 & 0.15 & 0.2 & 0.12 & 0.34 & 0.06 & 0.06 & 1.53 & 1.41 & 0.18 & $\begin{array}{ll}0.18 \\
\end{array}$ & 1.39 & 1.64 & 0.13 & 0.17 \\
\hline 40 & 0.24 & 0.58 & 0.06 & 0.08 & 0.15 & 0.31 & 0.07 & $\begin{array}{l}0.09 \\
\end{array}$ & 0.57 & 0.88 & 0.18 & 0.28 & 0.38 & 0.28 & 0.11 & 0.11 \\
\hline 41 & 0.45 & 0.52 & 0.1 & 0.12 & 0.33 & 0.46 & 0.13 & 0.18 & 1.05 & 1.59 & 0.21 & 0.24 & 0.92 & 0.91 & 0.08 & 0.09 \\
\hline 42 & 0.28 & 1.19 & 0.06 & 0.25 & 0.24 & 0.47 & 0.15 & 0.14 & 1.26 & 1.62 & 0.15 & 0.18 & 0.32 & 0.27 & 0.05 & 0.04 \\
\hline 44 & 1.06 & 1.2 & 0.18 & 0.22 & 0.27 & 0.97 & 0.1 & 0.18 & 0.24 & 0.29 & 0.1 & 0.15 & 0.45 & 0.31 & 0.09 & 0.1 \\
\hline 45 & 0.51 & 0.57 & 0.14 & 0.19 & 0.75 & 0.71 & 0.25 & 0.26 & 0.91 & 1.34 & 0.2 & 0.24 & 1.72 & 2.66 & 0.19 & 0.32 \\
\hline 46 & 0.33 & 0.43 & 0.06 & 0.06 & 0.14 & 1.75 & 0.07 & 0.22 & 0.8 & 2.37 & 0.13 & 0.19 & 0.59 & 1.09 & 0.1 & 0.12 \\
\hline 47 & 0.18 & 0.17 & 0.06 & 0.05 & 0.16 & 0.4 & 0.06 & 0.11 & 0.57 & 0.62 & 0.09 & 0.11 & 0.54 & 0.46 & 0.13 & 0.11 \\
\hline 48 & 0.41 & 0.5 & 0.14 & 0.16 & $\vec{\sigma} .3$ & 0.57 & $\begin{array}{lll}\exists & 0.11 & 0\end{array}$ & 0.21 & $\stackrel{\infty}{-\infty} \quad 0.71$ & $0 . \overrightarrow{53}$ & $\begin{array}{ll}\text { Na } & 0.0 \% \\
\end{array}$ & $0.2^{10}$ & $\begin{array}{ll}0.93 \\
\sim\end{array}$ & 0.82 & ले 0.19 & $0.19^{m}$ \\
\hline 49 & 0.42 & 0.63 & 0.1 & 0.16 & 0.27 & 0.52 & 0.13 & 0.19 & 0.44 & 0.89 & 0.08 & 0.12 & 0.78 & 0.73 & 0.17 & 0.17 \\
\hline 50 & 0.97 & 1.91 & 0.14 & 0.25 & 0.32 & 1.4 & 0.07 & 0.28 & 1.18 & 1.24 & 0.24 & 0.2 & 0.49 & 1.26 & 0.11 & 0.11 \\
\hline 51 & 0.35 & 0.45 & 0.15 & 0.2 & 0.12 & 0.15 & 0.07 & 0.07 & 1.24 & 1.22 & 0.21 & 0.2 & 2.29 & 4.77 & 0.16 & 0.3 \\
\hline 53 & 0.44 & 0.88 & 0.13 & 0.22 & 0.13 & 0.19 & 0.08 & 0.12 & 0.71 & 2.34 & 0.14 & 0.36 & 0.49 & 0.49 & 0.07 & 0.09 \\
\hline 54 & 0.34 & 0.42 & 0.07 & 0.08 & 0.21 & 0.78 & 0.08 & 0.13 & 0.36 & 0.52 & 0.07 & 0.09 & 1.27 & 1.16 & 0.1 & 0.11 \\
\hline 55 & 0.32 & 0.44 & 0.07 & 0.08 & 0.15 & 0.39 & 0.07 & 0.1 & 1.8 & 2.36 & 0.28 & 0.36 & 0.31 & 0.3 & 0.07 & 0.08 \\
\hline 56 & 0.74 & 0.68 & 0.08 & 0.08 & 0.21 & 0.98 & 0.1 & 0.17 & 1.43 & 2.1 & 0.25 & 0.28 & 0.33 & 0.51 & 0.1 & 0.1 \\
\hline
\end{tabular}

Table 4: The accuracy for each state for case forecasting using only visitor and local mobility, for the three machine learning models. 